\title{
Resonant third-order hyperpolarizabilities of large organic molecules
}

\author{
C. Maloney* and W. Blau \\ Department of Pure and Applied Physics, Trinity College, Dublin 2, Ireland
}

Received December 12, 1986; accepted February 24, 1987

\begin{abstract}
Resonant degenerate four-wave mixing in a number of infrared-absorbing dyes was studied with 160-psec pulses at $1.064 \mu \mathrm{m}$. Hyperpolarizabilities in the range of $10^{-44}$ to $10^{-42} \mathrm{~V}^{-2} \mathrm{~m}^{5}$ were observed. A theoretical model based on a two-band system is developed that explains the results and permits the prediction of hyperpolarizabilities from the linear spectroscopic properties.
\end{abstract}

\section{INTRODUCTION}

Third-order nonlinear optical processes have attracted considerable interest recently because of their potential applications in optical phase conjugation, optical computing, and dynamic holography and their power as a spectroscopic tool. ${ }^{1}$ Many of the studies, however, deal with inorganic semiconductors, photorefractive materials, and atomic vapors. ${ }^{2}$ The main research on organic materials in this area has centered on liquid crystals for which the nonlinearity is very large but also very slow ${ }^{3}$ and on semiconducting polymers that have a large very fast nonresonant nonlinearity. 4 The observation of the resonant susceptibility is usually occluded by a large thermally induced refractive-index change. ${ }^{5}$ Frequency-domain ${ }^{6}$ as well as picosecond and time-resolved studies ${ }^{7}$ of some large dye molecules show, however, the power of using cross-polarized pump and probe beams to prevent the setting up of a thermally induced phase grating and hence measuring resonant third-order hyperpolarizabilities. Appreciable nonlinearities can be observed, with the decay time of the nonlinearity being limited by the lifetime of the excited state, that is, of the order of picoseconds to nanoseconds for the samples used in this study.

\section{LASER-INDUCED ORIENTATION GRATINGS}

In degenerate four-wave mixing (DFWM; see Fig. 1) the nonlinear interaction is generally described by laser-induced gratings. With parallel-polarized beams, the probe beam $E_{3}$ and either of the pump beams $E_{1}$ and $E_{2}$ set up a spatial modulation of the absorption and/or the refractive index, termed concentration gratings. ${ }^{8}$ When this configuration is used, the intensity in the medium is modulated periodically in space, but the polarization direction is uniform everywhere.

In the experiments that follow, the probe wave beam is orthogonally polarized with respect to the two pump beams. The overlapping cross-polarized waves produce a polarization direction in the medium that is periodically modulated in space but has a uniform intensity distribution. By this technique concentration (and hence thermally induced) effects are eliminated, and intrinsic nonlinearities may be studied.
The spatial dependence of the polarization direction in the medium induced by orthogonally polarized overlapping waves is complicated. The interference pattern produced by overlap at an angle $\theta$ of the wave-front maxima of two fields $E_{1}$ and $E_{3}$ is shown in Fig. 2a. $E_{1}$ is linearly polarized perpendicular to the page ( $z$ direction). $E_{3}$ is linearly polarized in the plane of the page and has a polarization component $E_{3} \cos \theta$ along the $x$ direction.

At some particular instant in time, a wave front of the backward pump is incident at the plane indicated in the diagram.

The polarization directions at points $x_{1}$ to $x_{1} \pm \lambda / \sin \theta$ along this plane caused by the fields $E_{1}$ and $E_{3}$ are shown in Fig. 2b. This pattern is periodic of period $\Lambda=\lambda / \sin \theta$. The backward-pump wave $E_{2}$ thus encounters a spatially periodic polarization direction. At points $x_{1} \pm n \lambda / \sin \theta, n=0,1$, $2, \ldots$ the polarization is always linear at an angle $\alpha=\tan ^{-1}$ $\left|E_{1} / E_{3}\right|$ to the $z$ direction, since at these points the two waves $E_{1}$ and $E_{3}$ are in phase. Between these points, the field becomes somewhat elliptically polarized because of the phase difference between $E_{1}$ and $E_{3}$ and their differing magnitudes.

The effect on the medium is as follows: The excited-state concentration is uniform but has an orientation dependence proportional to

$$
\left|r_{12}(\theta, \phi) \cdot \hat{\epsilon}(r)\right|^{2},
$$

where $r_{12}(\theta, \phi)$ is the matrix element of the dipole moment $\mu_{12}$ of a molecule with coordinates $r, \theta$, and $\phi$ and $\hat{\epsilon}(r)$ is the resultant polarization vector at position $r$. The excitedstate concentration at points $x_{1} \pm n \lambda / \sin \theta$ is

$$
\Delta N \propto \cos ^{2} \beta,
$$

where $\beta$ is the angle between $\hat{\epsilon}(r)$ and the orientation of the molecule of interest. Between these points, the molecules aligned along the polarization experience preferential excitation.

Orientation gratings have been studied extensively in semiconductors. ${ }^{8,9}$ They arise through anisotropic-state filling where carriers are preferentially excited to energy states of a particular $k$ direction. Although the intensity throughout the interaction region of the medium is uniform, the orientation of the excited states is periodic in space. 

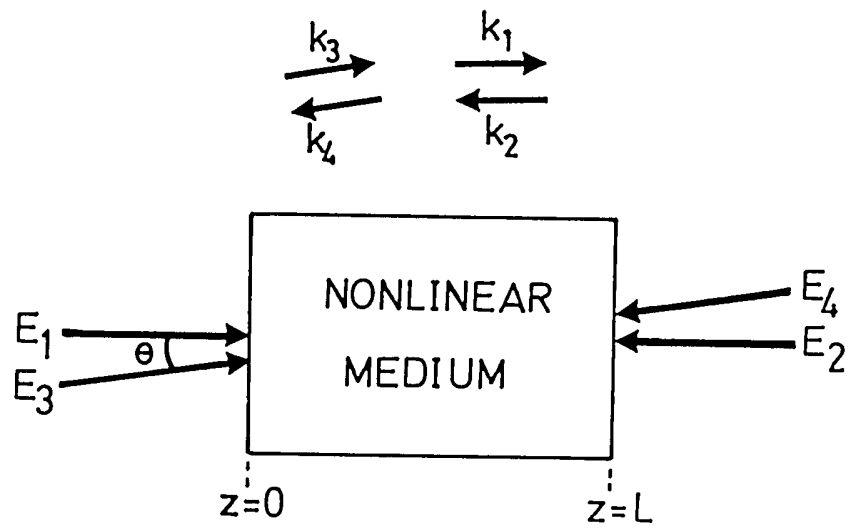

Fig. 1. Schematic illustrations of the DFWM geometry.

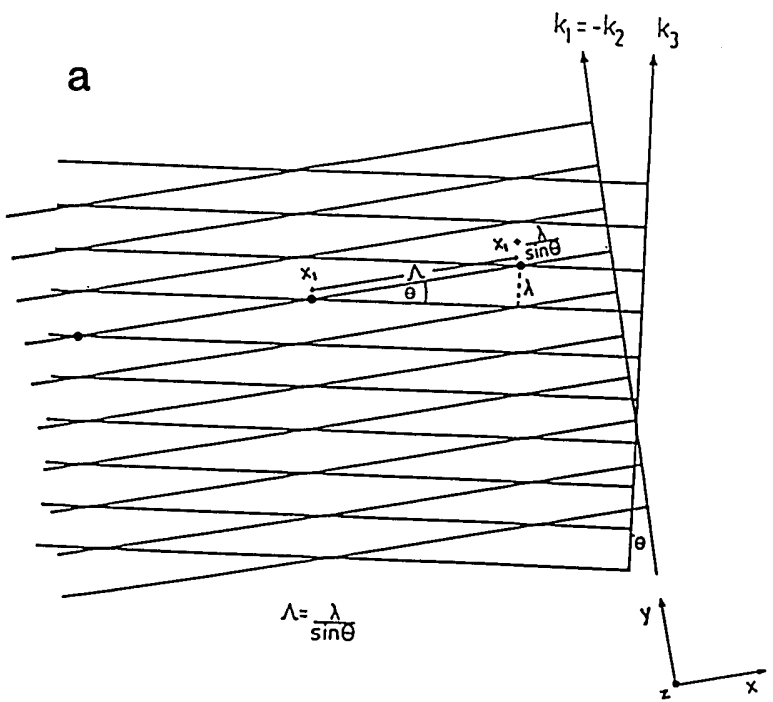

b

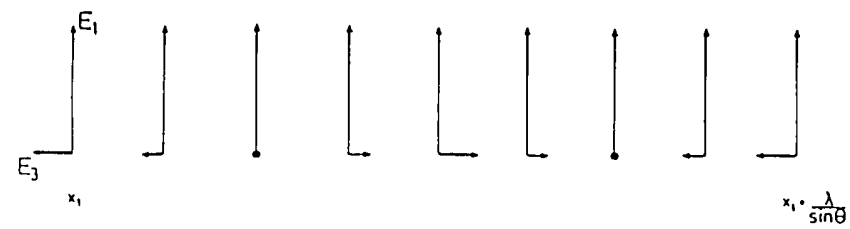

Fig. 2. a, Interference pattern created by overlap of pump wave $E_{1}$ and probe wave $E_{3}$ in the medium. b, Variation of the electric-field amplitudes and resultant polarization in the medium along the grating plane.

In liquids and liquid solutions two different processes can give rise to orientation gratings: alignment of the molecules along the field direction or distortion of the molecule's electron cloud by the field, both of which lead to a transient refractive-index grating in the material.

The first of these occurs in liquids composed of anisotropic molecules whose optical polarizabilities along different axes are unequal. In the absence of a field, the molecules of the liquid are randomly oriented, and its refractive index is isotropic. The periodic polarization field induces dipole moments that exert torques causing the molecule to be rotated into alignment with the field. Consequently, the refractive index becomes periodically modulated in space. The orientation time of the molecules may be estimated by using the Debye equation ${ }^{10}$

$$
\tau_{0}=\frac{4 \pi \eta a^{3}}{k T},
$$

where $\eta$ is the viscosity of the liquid, $a$ is the radius of the molecular volume, and $k$ and $T$ are Boltzmann's constant and the temperature, respectively. In $\mathrm{CS}_{2}$ this molecular Kerr effect forms the predominant contribution to its nonlinear index of refraction. ${ }^{10}$

In all materials, an electronic contribution to the nonlinear index of refraction can occur from an optically induced distortion of the electronic-cloud distribution. This effect is generally considerably smaller in magnitude than the molecular orientational Kerr effect. However, it can be magnified in organic systems containing long conjugated chains where delocalization of the $\pi$-electron cloud along the chain is possible. The effect is essentially instantaneous, with a time constant of $\sim 10^{-15} \mathrm{sec}$.

\section{EXPERIMENTS}

The experimental arrangement has been described in detail elsewhere. ${ }^{7}$ All the beams were derived from a single 160 psec laser pulse at $\lambda=1.064 \mu \mathrm{m}$. The samples were contained in $l=2$-mm-thick quartz cuvettes.

Each of the dyes investigated is listed in Fig. 3 together with the solvent and the spectral position of the absorption maximum. It was decided to work in liquid solutions, as problems with laser-induced damage are avoided. Of the

SAMPLE
CHEMICAL STRUCTURE
ABSORPTION

$\operatorname{MAX} .(\mu \mathrm{m})$

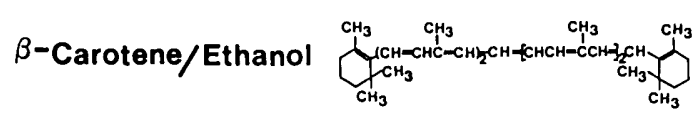

Nigrosine/Water

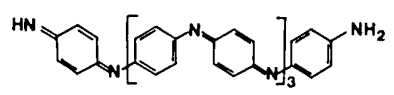

DTTC/Methanol
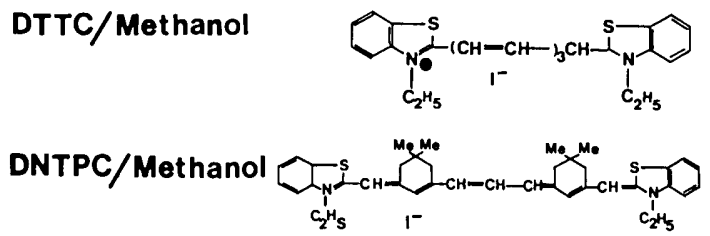

BDN/Toluene

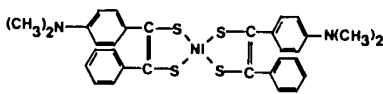

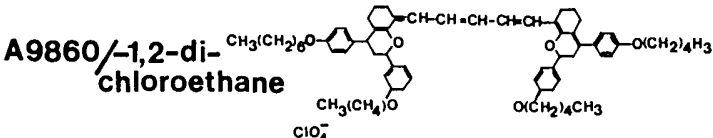

IR5/1,2- dichloroethane<smiles>OC1CC(O)C(O)C(O)O1</smiles>

\section{$\mathrm{ClO}_{4}^{-}$}

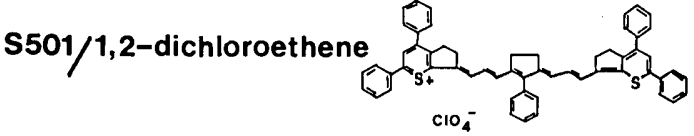

Fig. 3. Chemical structural formulas, solvents, and absorption maxima of the samples used. 

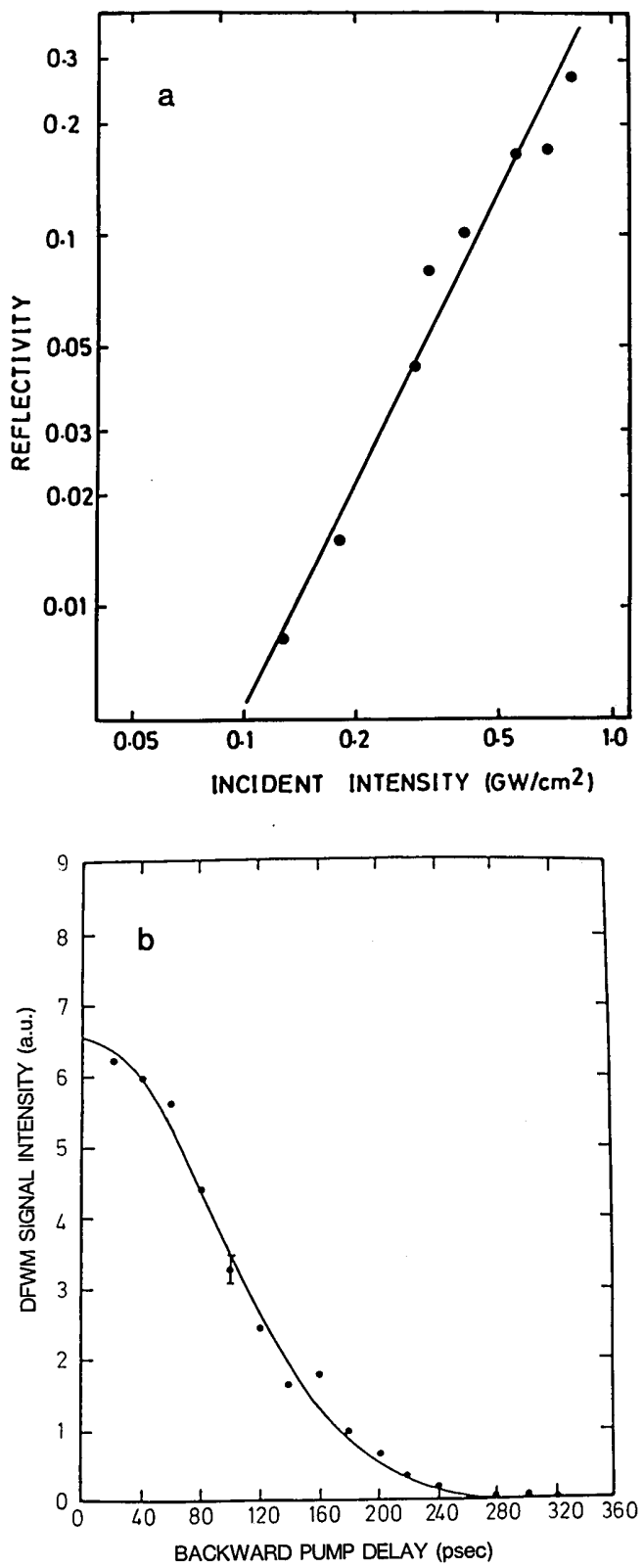

Fig. 4. a, DFWM signal peak reflectivity as a function of incident pump intensity; b, DFWM signal intensity as a function of the backward pump delay for a solution of S501 in 1,2 dichloroethane.

molecules chosen, only $\beta$-carotene has been shown to have a large nonresonant electronic nonlinearity that has been explained in terms of $\pi$-electron delocalization along its conjugated chain. ${ }^{11}$ Nigrosine is a short-chain substitute of the conducting polymer polyaniline and, as such, promises to have a large nonresonant susceptibility. ${ }^{12}$ Figure 4 shows the measured intensity dependence of the phase-conjugate reflectivity, $R_{\mathrm{PC}}$, with varying incident pump-pulse intensity for the dye S501. A square dependence is found. The decay of the nonlinear excitation was probed by temporally delaying the second pump pulse $E_{2}$. The decay in the case of S501 was found to be faster than the pulse width of the laser, in accordance with a measured excited-state lifetime of $17 \mathrm{psec}$ (Ref. 13) for this sample.

The third-order nonlinear susceptibility was calculated from $^{14}$

$$
\left|\chi_{x y y x}^{(3)}\right|=\frac{4 c^{2} \epsilon_{0} \sqrt{R_{\mathrm{PC}}} n^{2} \alpha}{3 \omega I T(1-T)}
$$

with sample transmission $T$, absorption coefficient $\alpha=$ $\ln (T) / l$, refractive index $n$, and laser frequency $\omega$.

In the case of low absorption, $T \rightarrow 1$, Eq. (4) can be reduced to

$$
\left.\right|_{x y y x} ^{(3)}=\frac{4 c^{2} \epsilon_{0} \sqrt{R_{\mathrm{PC}}} n^{2}}{3 \omega I l} .
$$

Table 1 gives a list of experimental parameters for each sample and the measured nonlinear susceptibilities.

For molecular systems the parameter describing the nonlinear response on a molecular scale is the hyperpolarizability. The third-order hyperpolarizability is related to $\chi^{(3)}$ by

$$
\gamma_{x y y x}=\frac{\chi_{x y y x}^{(3)}}{L_{L}{ }^{4} N_{0}},
$$

where $L_{L}$ is the Lorentz field factor accounting for the microscopic applied electric field and $N_{0}$ is the number density of dye molecules, related to the concentration $c$ by $N_{0}=N_{A} c$, with $N_{A}$ being Avogadro's number. For the calculations the formula for spherical molecules $L_{L}=(\epsilon(\omega)+2) / 3$ was used, which is sufficiently accurate to describe the studied molecules within the experimental errors. The conversion factor from SI to electrostatic units is

$$
\gamma_{\mathrm{esu}}=\gamma_{\mathrm{SI}} \times 7.16 \times 10^{13}
$$

Table 1 also lists the measured third-order hyperpolarizabilities. They clearly decrease with increasing detuning of the absorption maximum from the laser frequency, therefore pointing to a resonantly enhanced nonlinearity. In

\begin{tabular}{|c|c|c|c|c|c|c|c|}
\hline Dye & $T$ & $n$ & $R$ & $\chi_{x y y x}^{(3)}\left(\mathrm{m}^{2} / \mathrm{V}^{2}\right)$ & $L_{L}^{4}$ & $N_{0}\left(\mathrm{~m}^{-3}\right)$ & $\gamma_{x y y x}\left(\mathrm{~V}^{-2} \mathrm{~m}^{5}\right)$ \\
\hline$\beta$-Carotene & 1.0 & 1.3 & $<0.0010$ & $<0.2 \times 10^{-20}$ & 0.726 & $2 \times 10^{23}$ & $<1 \times 10^{-44}$ \\
\hline Nigrosine & 0.38 & 1.33 & 0.0056 & $2.62 \times 10^{-20}$ & 0.726 & $4.2 \times 10^{23}$ & $7.2 \times 10^{-44}$ \\
\hline DTTC & 1.0 & 1.3 & 0.0054 & $0.8 \times 10^{-20}$ & 0.726 & $2.5 \times 10^{23}$ & $4.9 \times 10^{-44}$ \\
\hline DNTPC & 0.94 & 1.3 & 0.0074 & $1.02 \times 10^{-20}$ & 0.726 & $4.3 \times 10^{22}$ & $3.27 \times 10^{-43}$ \\
\hline $\mathrm{BDN}$ & 0.53 & 1.49 & 0.011 & $1.7 \times 10^{-20}$ & 1.23 & $1.6 \times 10^{22}$ & $8.6 \times 10^{-43}$ \\
\hline A9860 & 0.65 & 1.45 & 0.023 & $1.75 \times 10^{-20}$ & 1.1 & $5.8 \times 10^{21}$ & $2.7 \times 10^{-42}$ \\
\hline IR5 & 0.55 & 1.45 & 0.021 & $2.13 \times 10^{-20}$ & 1.1 & $1 \times 10^{22}$ & $1.9 \times 10^{-42}$ \\
\hline S501 & 0.67 & 1.55 & 0.27 & $1.25 \times 10^{-19}$ & 1.65 & $5 \times 10^{21}$ & $1.27 \times 10^{-42}$ \\
\hline
\end{tabular}
what follows a theory is developed that seeks to explain the results at a more fundamental level.

Table 1. Solution Parameters and Measured Third-Order Nonlinearity for the Samples Used 


\section{THEORY}

The simplest but rather successful description of absorbing organic molecules is using a two-level (band) system. A precise treatment of the nonlinear interactions occurring must start with a density-matrix formulation and must take time ordering into account. A detailed treatment of this will be published by us elsewhere. In this paper we begin with the expression derived by Gower ${ }^{14}$ from time-ordered perturbation theory. This yields a third-order polarization induced in the medium of the form

$$
\begin{aligned}
P^{(3)}= & \frac{-2 i N_{0}}{(2 h)^{3}}\left|\mu_{12}\right|^{4} \epsilon_{1} \epsilon_{2} \epsilon_{3}{ }^{*}\left(\frac{1}{\phi_{1}}+\frac{1}{\phi_{2}}\right)\left[\frac{1}{\left(\Delta \omega-i \phi_{12}\right)}\right. \\
& \left.-\frac{1}{\left(\Delta \omega+i \phi_{21}\right)}\right] \frac{\exp \left[i\left(\omega t+k_{3} z\right)\right]}{\left(\Delta \omega+i \phi_{12}\right)}+\text { c.c. },
\end{aligned}
$$

where $\epsilon_{i}$ is the electric-field amplitude of beam $E_{i}, \Delta \omega$ is the detuning from resonance, and all other terms are as explained below or above.

This expression was originally derived for the third-order polarization giving rise to DFWM in narrow-band, two-level systems near resonance.

In such narrow-band systems as atomic sodium vapor, the energy-level populations decay radiatively at a rate $\phi_{1,2}=$ $\Gamma_{1,2}=1 / \tau_{\text {rad, }}$, and the total number of molecules contributes to the nonlinear polarization.

This is not the case in organic systems, in which the nonradiative decay rates $1 / \tau_{\text {nonrad }}$ are appreciable and often orders of magnitude greater than $1 / \tau_{\text {rad }}$. The effect of $\tau_{\text {nonrad }}$ is accounted for in the term

$$
\phi_{1,2}=\Gamma_{1,2}+\gamma_{1,2}^{c} \text {. }
$$

In the organic molecules

$$
\Gamma_{1,2}=1 / \tau_{\text {rad }}, \quad \gamma_{1,2}^{c}=1 / \tau_{\text {nonrad }},
$$

so the term $\left(1 / \phi_{1}+1 / \phi_{2}\right)$ in Eq. (8) becomes

$$
\begin{aligned}
1 /\left(\Gamma_{1}+\gamma_{1}^{c}\right)+1 /\left(\Gamma_{2}+\gamma_{2}^{c}\right) & =2 \tau_{F} \\
& =2 \phi_{F} \tau_{\mathrm{rad}},
\end{aligned}
$$

where $\phi_{F}$ is the fluorescence quantum yield. This implies that only molecules that decay radiatively contribute to nonlinear polarization. In most of the investigated inorganic molecules, the fluorescence quantum yield is as low as $\sim 10^{-4}$, such that the nonlinear polarization is considerably reduced. Consequently, organic molecules with high-fluorescence quantum yields are suited to efficient DFWM.

It should be mentioned that the induced nonlinearity de- cays with the coherence dephasing rate $\phi_{12}=1 / T_{2}=10^{14}$ $\sec ^{-1}$. As the excited-state population decays as $K_{\mathrm{rad}}=1 /$ $\tau_{\text {rad }}=10^{8} \mathrm{sec}^{-1}$, the maximum repetition rate is limited by $\tau_{\text {rad }}$ and not by $T_{2}$. Damage from high power absorption in the nonlinear material may, however, ultimately limit the repetition rate.

A further factor to be considered is that in liquid solutions the transition dipole moments of the solute molecules are randomly oriented. A simple geometrical calculation shows that the third-order hyperpolarizability components $\gamma_{x x x x}$ and $\gamma_{x y y x}$ in isotropic media are related by

$$
\gamma_{x y y x}=\gamma_{x x x x} / 3
$$

We can now write

$$
p^{(3)}(r, t)=\frac{3}{4} \epsilon_{0}\left(L_{L}^{4} N_{0} \gamma_{x y y x} \times 3\right) \frac{\epsilon_{1} \epsilon_{2} \epsilon_{3}{ }^{*}}{8} \exp \left[i\left(\omega t+k_{3} z\right)\right]+\text { c.c. }
$$

$$
\begin{aligned}
= & -\frac{2 i N_{0}}{8 h^{3}} \frac{\left|\mu_{12}\right|^{4}}{\left(\Delta \omega+i / T_{2}\right)} \phi_{F} 2 \tau_{\mathrm{rad}} \\
& \times\left[\frac{1}{\left(\Delta \omega-i T_{2}\right)}-\frac{1}{\left(\Delta \omega+i / T_{2}\right)}\right] \\
& \times \epsilon_{1} \epsilon_{2} \epsilon_{3}{ }^{*} \exp \left[i\left(\omega t+k_{3} z\right)\right]+\text { c.c. }
\end{aligned}
$$

Substituting 15

$$
\left|\mu_{12}\right|^{2}=\frac{3 \epsilon_{0} h c}{2 \omega_{0}^{3} \tau_{\mathrm{rad}} n^{3}}
$$

for the transition dipole moment and equating Eqs. (12) and (13) gives

$$
\left|\gamma_{x y y x}\right|=\frac{8 \epsilon_{0} c^{6} \phi_{F}}{h L_{L}{ }^{4} \omega_{0}{ }^{6} n^{6} \tau_{\text {rad }} T_{2}} \frac{1}{\left[\Delta \omega^{2}+\left(1 / T_{2}\right)^{2}\right]^{3 / 2}} .
$$

The relevant parameters are listed in Table 2. The values of $\gamma_{x y y x}$ predicted by the Eq. (15) are compared with measured values from Table 1 .

\begin{tabular}{|c|c|c|c|c|c|c|c|}
\hline Dye & $\omega\left(\sec ^{-1}\right)$ & $\Delta \omega\left(\sec ^{-1}\right)$ & $\tau_{\mathrm{rad}}$ (nsec) & $\tau_{f}(\mathrm{psec})$ & $T_{2}(\mathrm{fsec})$ & $\phi_{f}$ & $\gamma_{x y y x}\left(\mathrm{~V}^{-2} \mathrm{~m}^{5}\right)$ \\
\hline Nigrosine & $3.34 \times 10^{15}$ & $1.57 \times 10^{15}$ & 3 & - & 40 & $1.0 \times 10^{-4}$ & $3.25 \times 10^{-44}$ \\
\hline DTTC & $2.5 \times 10^{15}$ & $7.34 \times 10^{14}$ & 12 & 2000 & 18.7 & $1.67 \times 10^{-1}$ & $9.5 \times 10^{-43}$ \\
\hline DNTPC & $1.89 \times 10^{15}$ & $1.2 \times 10^{14}$ & 54 & - & 7.6 & $1.0 \times 10^{-4}$ & $1.16 \times 10^{-43}$ \\
\hline BDN & $1.81 \times 10^{15}$ & $0.41 \times 10^{14}$ & 56 & 5600 & 14.8 & $1.0 \times 10^{-1}$ & $2.7 \times 10^{-43}$ \\
\hline A9860 & $1.76 \times 10^{15}$ & - & 28 & 7.0 & 18.1 & $2.5 \times 10^{-4}$ & $4.9 \times 10^{-42}$ \\
\hline IR5 & $1.73 \times 10^{15}$ & - & 37 & 2.7 & 18.5 & $7.3 \times 10^{-5}$ & $1.26 \times 10^{-42}$ \\
\hline S501 & $1.26 \times 10^{15}$ & - & 90 & 17 & 15.3 & $1.89 \times 10^{-4}$ & $2.56 \times 10^{-42}$ \\
\hline
\end{tabular}

Values for $\tau_{\text {rad }}$ were calculated from absorption and fluorescence spectra by using the Strickler-Berg expression ${ }^{16}$

$$
\tau_{\mathrm{rad}}=8 \pi c n^{2} \frac{\int E(\lambda) \lambda^{4} \mathrm{~d} \lambda}{\int E(\lambda) \lambda \mathrm{d} \lambda} \int \frac{\sigma(\lambda)}{\lambda} \mathrm{d} \lambda,
$$

with $E(\lambda)$ being the fluorescence spectrum and $\sigma$ the absorption cross section. Values for $T_{2}$ were estimated from the inverse linewidth of the absorption spectra $\left(T_{2} \sim 1 / \Delta \nu_{a}\right)$ and agree to within 1 order of magnitude with direct femtosecond measurements. ${ }^{17}$

Agreement between experiment and theory is remarkably

Table 2. Parameters Used in Calculation of the Third-Order Hyperpolarizabilities of the Dye Samples and Third-Order Hyperpolarizabilities Calculated Using Eq. (15) 
good, considering the number of variables to be measured experimentally with errors ranging from 5 to $20 \%$. In addition, the derivation of the expression for $\gamma$ is based on a quantum-mechanical approach, and there are no adjustable parameters. Of significance is the fact that the theoretical values of $\gamma$ for dyes whose absorption maxima fall to the long-wavelength side of $1.064 \mu \mathrm{m}$ agree well with experimentally measured values if $\Delta \omega$ is taken to be zero. This indicates simply that, on the time scale of the total interactions taking place within $T_{2}$, the dye absorption appears inhomogeneously broadened.

\section{CONCLUSIONS}

We have measured the third-order hyperpolarizabilities of a range of infrared-absorbing dye molecules. A theoretical model was developed that describes the nonlinear interaction by using a two-level system. The agreement between theory and experiments is remarkably good, such that a prediction of third-order nonlinearities from the theory is possible to better than $25 \%$ from absorption, fluorescence, and quantum yield data only.

The results, both experimental and theoretical, suggest that near-infrared-absorbing organic materials, because of their large nonlinear optical susceptibilities and fast response and decay times, should be strong candidates as materials for all-optical signal-processing devices at wavelengths between 1.0 and $1.6 \mu \mathrm{m}$. Incorporation of these molecules into thin-film structures such as Langmuir-Blodgett multilayers would enhance the susceptibilities further, thus rendering them more compatible with semiconductor lasers and more practical as materials for such devices.

\section{ACKNOWLEDGMENTS}

Donations of dye samples by K. H. Drexhage, UniversitätGesamthochschule Siegen, and A. Penzkofer, Universität Regensburg, are gratefully acknowledged. The authors thank British Petroleum Venture Research, Guinness and
Co. Ltd., the Investment Bank of Ireland, and the Directorate for Scientific Research DGX11/B/2 of the European Economic Communities for their financial support.

* Present address, Department of Physics and Astronomy, Dartmouth College, Hanover, New Hampshire 03755.

\section{REFERENCES}

1. Y. R. Shen, The Principles of Nonlinear Optics (Wiley, New York, 1984).

2. R. A. Fisher, ed., Optical Phase Conjugation (Academic, New York, 1983).

3. I.-C. Khoo, IEEE J. Quantum Electron. QE-22, 1268 (1986).

4. C. Flytzanis, in Nonlinear Optical Properties of Organic and Polymeric Materials, D. J. Williams, ed., ACS Symposium Series No. 233 (American Chemical Society, Washington, D.C., 1983), pp. 167-185.

5. J. O. Tocho, W. Sibbett, and D. J. Bradley, Opt. Commun. 37, 67 (1981).

6. R. Trebino, C. E. Barker, and A. E. Siegman, IEEE J. Quantum Electron. QE-22, 1413 (1986).

7. C. Maloney, W. Blau, and K. H. Drexhage, Opt. Lett. 11, 434 (1986).

8. A. L. Smith, T. F. Boggess, B. S. Wherrett, G. P. Perryman, and A. Miller, Phys. Rev. Lett. 49, 933 (1982).

9. J. L. Oudar, I. Abram, and C. Minot, Appl. Phys. Lett. 44, 689 (1984).

10. P.D. Maker, R. W. Terhune, and C. M. Savage, Phys. Rev. Lett. 12, 507 (1964).

11. J. P. Hermann, D. Ricard, and J. Ducuing, Appl. Phys. Lett. 23, 178 (1973).

12. W. R. Salanek, I. Lundström, B. Liedberg, M. A. Hasan, R. Erlandsson, P. Konradson, A. G. MacDiarmid, and N. L. D. Somasiri, in Electronic Properties of Polymers and Related Components, H. Kuzmany, M. Mehring, and S. Roth, eds., Vol. 63 of Springer Series in Solid State Sciences (Springer-Verlag, Berlin, 1985), pp. 218-222.

13. H. J. Polland, T. Elsaesser, A. Seilmeier, W. Kaiser, M. Kussler, N. J. Marx, B. Sens, and K. H. Drexhage, Appl. Phys. B 32, 53 (1983).

14. M. C. Gower, IEEE J. Quantum Electron. QE-21, 182 (1985).

15. W. Demtroeder, Laser Spectroscopy, Vol. 5 of Springer Series in Physics (Springer-Verlag, Berlin, 1982).

16. S. J. Strickler and R. A. Berg, J. Chem. Phys. 37, (1962) 814.

17. A. M. Weiner and E. P. Ippen, Opt. Lett. 9, 53 (1984).

\section{Maloney}

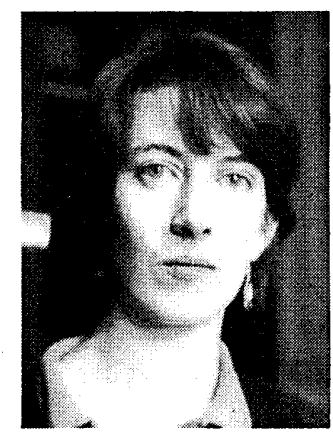

C. Maloney was born in Dublin, Ireland, in 1962. She received the bachelor's degree in physics and mathematics from Trinity College, Dublin, in 1982 and the $\mathrm{Ph} . \mathrm{D}$. degree in physics from Trinity College in 1986 for work on nonlinear optics in organic materials. She is now at the Department of Physics and Astronomy, Dartmouth College, Hanover, studying exciton dynamics in semiconducting superlattices and quantum wells.

\section{W. Blau}

W. Blau was born in 1954 in Regensburg, Federal Republic of Germany. He did his undergraduate and graduate studies in physics at the University of Regensburg, obtaining the degrees of Diplomphysiker and the doctoral Dr. rer. nat. in 1979 and 1983, respectively, for his work on picosecond solid-state lasers and spectroscopy. $\mathrm{He}$ joined Trinity College, Dublin, in 1983 as a British Petroleum Venture Research Fellow with D. J. Bradley. Since 1985 he has held a Lectureship in the Science of Materials/Physics in Trinity College. His main research topics at present are nonlinear optical properties of organic dyes, conducting polymers, organic metals, and low-dimensional semiconductor structures. Additional interests include laser-induced photolithography and the interaction of UV light with DNA. Dr. Blau is a member of the Optical Society of America, the Institute of Physics, and the German Physical Society. 